\title{
Outcomes and toxicity of radiotherapy for refractory bone and soft tissue sarcomas
}

\author{
HIROSHI DOI ${ }^{1,2}$, RYOONG-JIN OH ${ }^{1}$, HIDEHARU MIURA ${ }^{1}$, \\ NORIHISA MASAI ${ }^{1}$, HIROYA SHIOMI $^{1}$ and TOSHIHIKO INOUE ${ }^{1}$ \\ ${ }^{1}$ Miyakojima IGRT Clinic, Osaka 534-0021; ${ }^{2}$ Department of Radiology, \\ Hyogo College of Medicine, Nishinomiya, Hyogo 663-8501, Japan
}

Received April 14,2015; Accepted September 18, 2015

DOI: $10.3892 / \mathrm{mco} .2015 .654$

\begin{abstract}
Surgical resection is a well-established treatment option for sarcoma. However, anatomical barriers often hamper radical surgical procedures. The treatment of unresectable sarcoma, including local or distant failures following initial treatment, is challenging. The aim of the present study was to analyze the outcome of radiotherapy (RT) for refractory sarcoma, including unresectable, metastatic and recurrent disease, following radical treatment. We retrospectively reviewed a total of 67 tumors in 28 patients who were treated with RT between 2007 and 2014. Clinical target volume (CTV) was generally not defined in a preventive manner; therefore, in the majority of the cases, CTV equaled the gross tumor volume. The total delivered dose, number of fractions and biological equivalent dose were 52 (range, 40-69), 10 (range, 4-24) and 92.2 (range, 56-119.6) Gy, respectively. Only 1 patient developed local failure, with a median follow-up of 11 months (range, 1-59 months). Therefore, the 12-month progression-free survival rate for 67 sites was $96.8 \%$. The overall survival rates at 12 and 36 months were 75.8 and $30.2 \%$, respectively. A total of 2 patients developed grade $>2$ toxicities, including grade 3 mucositis and grade 4 pericardial effusion. Our results demonstrated that radical RT using modern techniques is highly feasible, achieves excellent local control, and may be an effective treatment option for refractory sarcoma.
\end{abstract}

\section{Introduction}

Surgical removal, requiring wide excision with negative margins, is a standard treatment option for sarcoma $(1,2)$.

Correspondence to: Dr Hiroshi Doi, Miyakojima IGRT Clinic, 1-16-22 Miyakojima Hondori, Miyakojima-ku, Osaka 534-0021, Japan E-mail: h-doi@hyo-med.ac.jp

Key words: stereotactic body radiation therapy, intensity-modulated radiation therapy, stereotactic ablative radiotherapy, radiotherapy, stereotactic radiotherapy, unresectable sarcoma, metastatic sarcoma, sarcoma
However, several factors, including anatomical barriers, make radical surgical procedures more challenging.

Approximately $50 \%$ of patients with sarcoma develop metastases during the course of the disease (3). However, the treatment for metastatic sarcoma is currently insufficient and represents a challenge (1-4).

Solid tumor metastases are considered non-curable. In addition, metastatic disease is associated with poor outcome, as few patients achieve durable disease control (4). A number of sarcomas exhibit a unique biological preference for the lungs, which are often the only sites of metastatic disease. Therefore, controlling these specific sites of progression may improve survival (3).

In recent years, the use of local treatment for sarcoma has increased. Historically, surgical resection of metastases has been primarily investigated in young patients. There is also evidence supporting the benefit of surgery for the treatment of metastases from soft tissue sarcomas, with surgery improving survival in selected cases (5). This aggressive surgical approach was retrospectively assessed for several sarcoma subtypes, providing an evidential foundation for ablative techniques. Surgery currently has the highest level of evidential support for the ablation of limited metastases, due to reports of patients cured from metastases (6).

Radiotherapy (RT) is integral in multimodal treatment and palliative care $(1,2)$. The use of RT is more generally accepted for the treatment of borderline resectable disease. Although improvements in RT techniques have reduced the exposure of the surrounding organs at risk (OARs), the effectiveness of RT in treating unresectable sarcoma, including metastases and local failure following initial treatment, remains largely unknown (7-13).

The aim of the present study was to analyze the outcomes following RT for sarcoma in the refractory setting, including unresectable, metastatic and recurrent disease following radical treatment.

\section{Patients and methods}

Patients. Between April, 2007 and March, 2014, a total of 28 patients who were treated with RT at the Miyakojima IGRT Clinic (Osaka, Japan) were found to be eligible for this study. The patient medical records were retrospectively reviewed for 
data analysis. The median age of the patients was 59.5 years (range, 19-81 years). The median follow-up was 11 months (range, 1-59 months) for a total of 67 sites treated with RT and 15.5 months (range, 2-59 months) for all patients. All the patients had histological evidence of sarcoma and all the tumors were deemed medically inoperable. The median duration between pathological confirmation of sarcoma and RT was 25 months (range. 1-341 months). A total of 15 (53.6\%) patients had been previously treated with RT, including proton and carbon ion RT. A total of 7 (25.0\%) patients received RT at our institute for in-field relapse following previous RT. The patient characteristics are summarized in Table I. A total of 67 gross tumor volumes (GTVs) were treated. All the treated patients signed an informed consent form prior to RT. Patients without macroscopically defined tumors following surgery were excluded from our analysis.

$R T$. Images for treatment planning were collected using BrightSpeed $^{\mathrm{TM}}$ (GE Healthcare, Milwaukee, WI, USA) for computed tomography (CT) simulation and Signa $\mathrm{HDx}^{\mathrm{TM}}$ (GE Healthcare) for magnetic resonance imaging (MRI) simulation. In the majority of the cases, patients underwent both CT imaging and MRI. The images were subsequently fused using treatment planning software. During simulation, all the patients receiving $\mathrm{RT}$ for tumors in the thoracic or abdominal area were immobilized with a vacuum bag in the supine arms-up position and underwent 4-dimensional (4D) CT simulation, obtaining $1.25-\mathrm{mm}$ slices with the free-breathing approach. Binning of corresponding 4D-CT images from all couch positions into different volumes generated actual volumetric spatiotemporal anatomical data. Ultimately, the CT data for target volume definition sets were generated according to the four respiratory cycle phases $(0,25,50$ and $75 \%)$, as previously described (14).

Clinical target volume (CTV) was generally not defined in a preventive manner. Therefore, the planning target volume (PTV) was obtained by expanding GTV. CTV equaled GTV in all but 2 cases. In 1 patient with a left coxal bone (acetabulum) metastasis from angiosarcoma of the left breast, the CTV was determined by adding a regional margin of the bone to the GTV, which was defined by CT and MRI. In another patient, 3 GTVs (lymph node failures in the right supraclavicular area, mediastinum and left pulmonary hilum resulting from carcinosarcoma of the uterine body) were treated as a single CTV including the regional lymph nodes. To account for tumor motion, an internal target volume (ITV) was generated by contouring. To create the PTV, an average margin of $5 \mathrm{~mm}$ (range, $2-8 \mathrm{~mm}$ ) was added to each ITV. In 1 case we treated 2 GTVs ( 1 in the left adductor brevis muscle and 1 in the left ischial bone) as a single PTV; this reduced the total number of PTV for each site from 67 to 64 .

BrainSCAN $^{\mathrm{TM}}$ and iPLAN RT Dose, version 4.1.2 $2^{\mathrm{TM}}$ (BrainLab AG, Feldkirchen, Germany) were used to plan treatment and prescribe doses with $95 \%$ coverage of the PTV using conformal beams or intensity-modulated maps. Dose calculation was performed with the pencil beam (PB) algorithm (BrainSCAN) or the Monte Carlo (MC) algorithm (iPlan $\mathrm{RT}$ ). In cases where both algorithms were available in a treatment plan, we presented the dose-volume histogram (DVH) as an MC algorithm. GTV and the biological equivalent dose
Table I. Patient characteristics $(n=28)$.

\begin{tabular}{lc}
\hline Characteristics & No. $(\%)$ \\
\hline Gender & \\
Male & $14(50.0)$ \\
Female & $14(50.0)$ \\
ECOG-PS score & \\
0 & $6(21.43)$ \\
1 & $15(53.57)$ \\
2 & $7(25.00)$ \\
KPS score & \\
100 & $6(21.43)$ \\
90 & $12(42.86)$ \\
80 & $3(10.71)$ \\
70 & $5(17.86)$ \\
60 & $1(3.57)$ \\
50 & $1(3.57)$
\end{tabular}

Primary site

Extremities

$8(28.57)$

Head and neck

$5(17.86)$

Retroperitoneum

4 (14.29)

Uterus

$4(14.29)$

Scapula

$1(3.57)$

Breast

$1(3.57)$

Subcutaneous tissue

$1(3.57)$

Cervical spine

$1(3.57)$

Axilla

$1(3.57)$

Ilium

$1(3.57)$

Tunica vaginalis testis

$1(3.57)$

Pathological diagnosis

Leiomyosarcoma

$7(25.00)$

Chondrosarcoma

$3(10.72)$

Osteosarcoma

3 (10.72)

Angiosarcoma

2 (7.14)

2 (7.14)

2 (7.14)

2 (7.14)

1 (3.57)

1 (3.57)

$1(3.57)$

$1(3.57)$

$1(3.57)$

$1(3.57)$

1 (3.57)

Sarcoma $^{\mathrm{a}}$

32 (47.76)

21

Lung

3

Thoracic wall

2

Supraclavicular node 2

Mediastinal node $\quad 2$

Hilar lymph node $\quad 1$

Clavicle 1

Rib

Sternum

1

1

Abdomen and pelvis 
Table I. Continued.

\begin{tabular}{|c|c|}
\hline Characteristics & No. $(\%)$ \\
\hline Iliac bone & 4 \\
\hline Retroperitoneum & 3 \\
\hline Ischial bone & 2 \\
\hline Pubis & 1 \\
\hline Coxal bone & 1 \\
\hline Presacral region & 1 \\
\hline Anal region & 1 \\
\hline Spine & $14(20.90)$ \\
\hline Thoracic vertebra & 6 \\
\hline Cervical vertebra & 5 \\
\hline Lumbar spine & 1 \\
\hline Sacral bone & 2 \\
\hline Head and neck & $6(8.96)$ \\
\hline Skull bone & 2 \\
\hline Skull base & 1 \\
\hline \multicolumn{2}{|l|}{ Semispinal muscle } \\
\hline Cervical node & 1 \\
\hline Maxillary bone & 1 \\
\hline Extremities & $2(2.99)$ \\
\hline Adductor brevis muscle & 1 \\
\hline Femur & 1 \\
\hline \multicolumn{2}{|l|}{ Tumor status } \\
\hline Unresectable primary disease & $2(2.99)$ \\
\hline Local relapse after initial treatment & $5(7.46)$ \\
\hline Regional lymph node metastasis & $2(2.99)$ \\
\hline Distant metastasis & $58(86.57)$ \\
\hline
\end{tabular}

(BED) were calculated for each individual lesion. The BED was calculated using the linear quadratic (LQ) formula:

$$
\mathrm{BED}=\mathrm{nd}[1+\mathrm{d} / \alpha / \beta] \text {, }
$$

assuming an $\alpha / \beta$ ratio of 10 (BED10) for the tumors and 3 (BED3) for normal tissues (n, number of fractions and $\mathrm{d}$, dose per fraction).

A summary of RT is shown in Table II and the BED10 of RT delivered for each GTV is shown in Table III. Between April, 2007 and June, 2012, intensity-modulated RT (IMRT) and conformal beams were used for 26 and 16 treatments, respectively. The remaining 22 treatments from July, 2012 to March, 2014 were performed using IMRT. The averages for delivered doses to the D95 of the PTV were $97.7 \%$ (range, 82.3-99.4\%) and 92.1\% (range, 73.8-97.9\%) in 30 PTVs using the PB algorithm and in 34 PTVs using the MC algorithm, respectively.

Using the PB algorithm, the average for delivered doses to the D99 of 23 GTVs in 8 treatment plans (6 for lung tumors) using conformal beams was $100.1 \%$ (range, 83.6-103.0\%). Dose calculations using the MC algorithm for treatment plans of lung tumors, including 8 using conformal beams and
Table II. Summary of radiotherapy.

\begin{tabular}{lc}
\hline Characteristics & Values (range) \\
\hline Total dose, Gy & $52(40-69)$ \\
Number of fractions & $10(4-24)$ \\
Fraction size, Gy & $5.5(2.8-13)$ \\
BED10, Gy & $92.2(56-119.6)$ \\
BED3, Gy & $168.8(93.3-277.3)$ \\
Treatment term, days & $12(3-34)$ \\
GTV, $\mathrm{cm}^{3}$ & $33.8(0.2-1006.9)$ \\
PTV, $\mathrm{cm}^{3}$ & $61.9(6.0-1384.8)$
\end{tabular}

BED, biological equivalent dose; GTV, gross tumor volume; PTV, planning target volume.

Table III. Number of tumors according to BED10 delivered.

\begin{tabular}{lc}
\hline BED10, Gy & No. of tumors \\
\hline$<70$ & 4 \\
$\geq 70,<80$ & 13 \\
$\geq 80,<90$ & 15 \\
$\geq 90,<100$ & 9 \\
$\geq 100,<110$ & 24 \\
$\geq 110,<120$ & 2 \\
Total & 67 \\
\hline BED, biological equivalent dose. & \\
\hline
\end{tabular}

26 using IMRT, revealed an average delivered dose to the D99 of 28 GTVs of $98.6 \%$ (range, $85.4-102.5 \%$ ).

The planned RT was delivered using a 6-MV X-ray Novalis unit $^{\mathrm{TM}}$ (BrainLab AG) on immobilization devices including Vac-Lok cushions ${ }^{\mathrm{TM}}$ and Thermoplastics (CIVCO Medical Solutions, Kalona, IA, USA); ExacTrac ${ }^{\mathrm{TM}}$ X-ray positioning system and 6-axis robotic couch (BrainLab AG) were used as an image-guided RT system.

Follow-up. Local control was defined as the time between the first day of RT and detection of local failure, or last follow-up. Local failures were identified by experienced physicians using physical examination, CT imaging and MRI. Overall survival (OS) was defined as the time between the first day of RT and death, or last follow-up. Toxicity was graded using the National Cancer Institute Common Terminology Criteria for Adverse Events, version 3.0 (15).

Statistical analysis. Continuous variables are expressed as medians (range) unless otherwise indicated. The interval between events was calculated from the first day of RT to the day of confirmation of an event. Cumulative local control and OS estimates were calculated using the Kaplan-Meier method and statistical differences were evaluated by the log-rank (Mantel-Cox) test. $\mathrm{P}<0.05$ was considered to indicate a statistically significant difference. All the analyses were performed 
Table IV. Univariate analysis for survival.

\begin{tabular}{|c|c|c|c|c|}
\hline Parameters & No. of patients & 2-year-OS (\%) & HR $(95 \%$ CI) & P-value \\
\hline \multicolumn{5}{|l|}{ Age, years } \\
\hline$<65$ & 21 & 53.4 & \multirow[t]{2}{*}{$1.29(0.37-4.40)$} & \multirow[t]{2}{*}{0.696} \\
\hline$\geq 65$ & 7 & 68.6 & & \\
\hline \multicolumn{5}{|l|}{ Gender } \\
\hline Male & 14 & 53.4 & \multirow[t]{2}{*}{$2.00(0.66-6.59)$} & \multirow[t]{2}{*}{0.218} \\
\hline Female & 14 & 62.9 & & \\
\hline \multicolumn{5}{|l|}{ ECOG-PS score } \\
\hline 0 & 6 & 80.0 & \multirow[t]{2}{*}{$0.22(0.10-1.19)$} & \multirow[t]{2}{*}{0.100} \\
\hline 1,2 & 22 & 50.6 & & \\
\hline \multicolumn{5}{|l|}{ KPS score } \\
\hline 90,100 & 18 & 54.6 & \multirow[t]{2}{*}{$0.47(0.13-1.45)$} & \multirow[t]{2}{*}{0.179} \\
\hline$\leq 80$ & 10 & 64.0 & & \\
\hline \multicolumn{5}{|l|}{ CCI } \\
\hline$\leq 7$ & 9 & 71.1 & \multirow[t]{2}{*}{$1.19(0.37-3.87)$} & \multirow[t]{2}{*}{0.761} \\
\hline$>7$ & 19 & 52.0 & & \\
\hline \multicolumn{5}{|c|}{ Age-adjusted CCI } \\
\hline$\leq 7$ & 7 & 57.1 & \multirow[t]{2}{*}{$1.30(0.38-4.71)$} & \multirow[t]{2}{*}{0.661} \\
\hline$>7$ & 21 & 58.3 & & \\
\hline \multicolumn{5}{|l|}{ Metastatic status } \\
\hline Single site & 8 & 87.5 & \multirow[t]{2}{*}{$0.29(0.15-0.84)$} & \multirow[t]{2}{*}{0.024} \\
\hline Multiple sites & 20 & 20.0 & & \\
\hline \multicolumn{5}{|l|}{ Treatment site } \\
\hline Primary & 7 & 57.1 & \multirow[t]{2}{*}{$2.40(0.78-11.53)$} & \multirow[t]{2}{*}{0.116} \\
\hline Metastatic & 21 & 59.9 & & \\
\hline
\end{tabular}

OS, overall survival; HR, hazard ratio; CI, confidence interval; ECOG-PS, Eastern Cooperative Oncology Group performance status; KPS, Karnofsky performance status; CCI, Charlson Comorbidity Index.

using the GraphPad Prism software, version 6.0b (GraphPad Software, Inc., San Diego, CA, USA).

\section{Results}

Local failure and survival. There was no reported local failure during follow-up, except for 1 patient (Fig. 1). This particular myxofibrosarcoma patient received $66 \mathrm{~Gy}$ in 22 fractions for lymphadenopathy in the left neck and developed local failure 10 months after the initiation of RT. OS is shown in Fig. 2. The OS rate was 75.8 and $30.2 \%$ at 12 and 36 months, respectively.

Univariate analyses of various factors were conducted to identify associations with survival (Table IV). According to the results, patients with a single viable sarcoma site exhibited significantly better OS compared with patients with multiple disease sites at the initiation of RT $(\mathrm{P}=0.024)$ (Fig. 3). The 12 -month disease-free survival rate was $45 \%$ in 8 patients who had a single viable tumor site (Fig. 4).

Toxicity. A total of 9 treatment-related toxicities developed in 6 patients. A total of 5 cases of grade 1 dermatitis, 1 case of grade 1 esophagitis and 1 case of grade 2 pneumonitis were identified. Grade 3 mucositis developed in 1 patient who

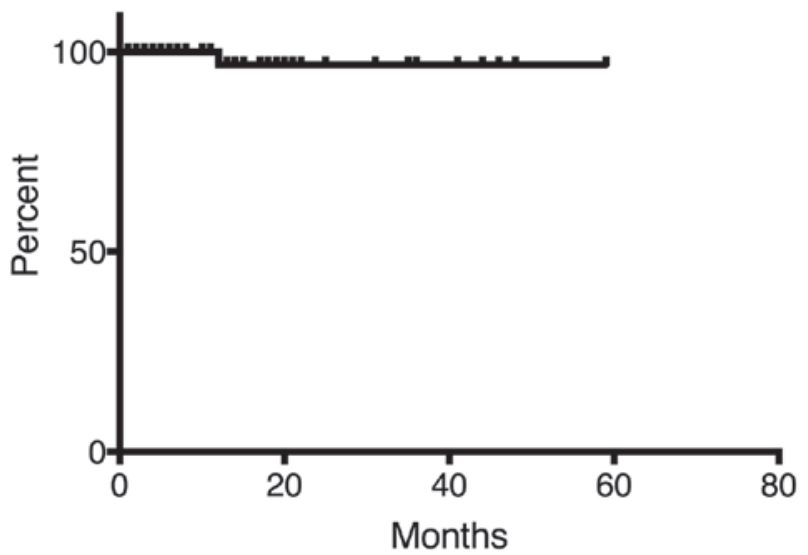

Figure 1. Progression-free survival (PFS) for treated sites. The PFS rate for 67 sites was $96.8 \%$ at 12 months.

underwent irradiation of the left maxillary bone; 1 patient, who received a single treatment of $60 \mathrm{~Gy}$ in 20 fractions to the left pulmonary hilum, mediastinum and right supraclavicular area, developed grade 4 pericardial effusion requiring drainage at 2 months following RT. To evaluate the effect of 


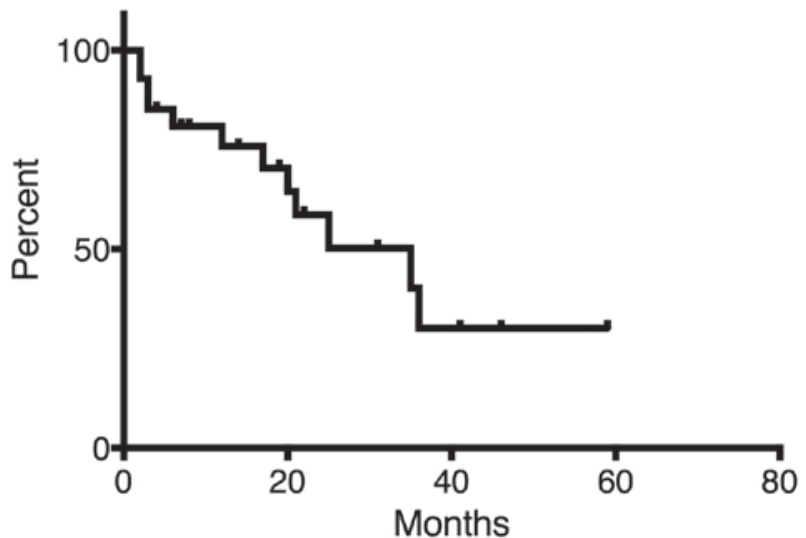

Figure 2. Overall survival (OS) for all patients. The median OS for the 28 patients was 35 months. The OS rate at 12 and 36 months was 75.8 and $30.2 \%$, respectively.

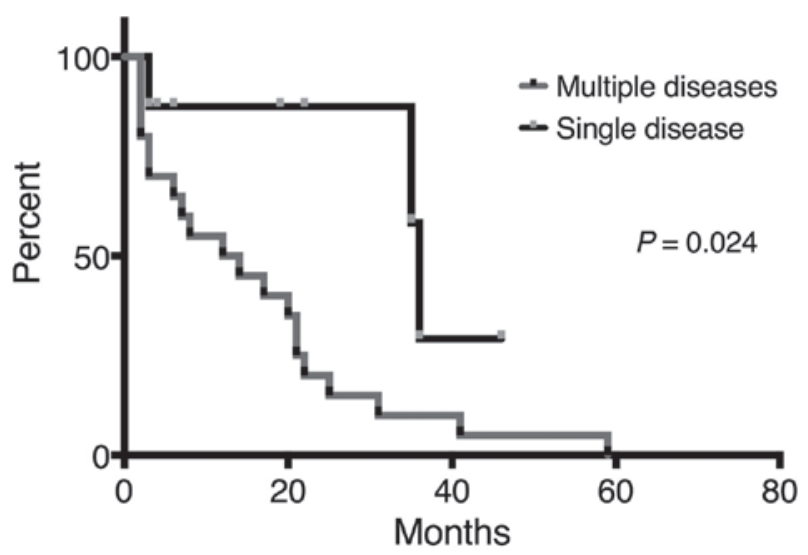

Figure 3. Overall survival (OS) of patients based on disease condition (single viable disease or multiple diseases). The median survival and OS rate at 22 months were 36 months and $87.5 \%$, respectively, in patients with a single viable disease, and 13 months and $20.0 \%$, respectively, in patients with multiple diseases.

RT on pericardial effusion, we analyzed the DVH of the heart in this patient. Dose distributions were corrected to the equivalent dose in 2-Gy fractions (EQD2) using the LQ model for an $\alpha / \beta$ value of $3 \mathrm{~Gy}$. A total of $6 \%$ of the heart volume $(1,030 \mathrm{ml})$ received $\geq 30$ Gy (EQD2). The maximum and mean doses for the heart were 104 and $14.4 \%$ of the prescribed dose, respectively. No patients developed RT-related late adverse events.

\section{Discussion}

The treatment of refractory sarcomas, including metastases, recurrent sites and unresectable sites, is challenging. Chemotherapy, such as doxorubicin, is commonly used for metastatic disease, although its efficacy is insufficient $(1,2,4)$. For patients with metastases limited to a single organ, surgery is historically performed to improve local control and survival. However, radical surgery is not always possible due to several factors. Recent reports describing alternative treatment options, including radiofrequency ablation and RT with modern modalities, show improvements in local control that may prolong survival in sarcoma patients (6,10-13). Consistent

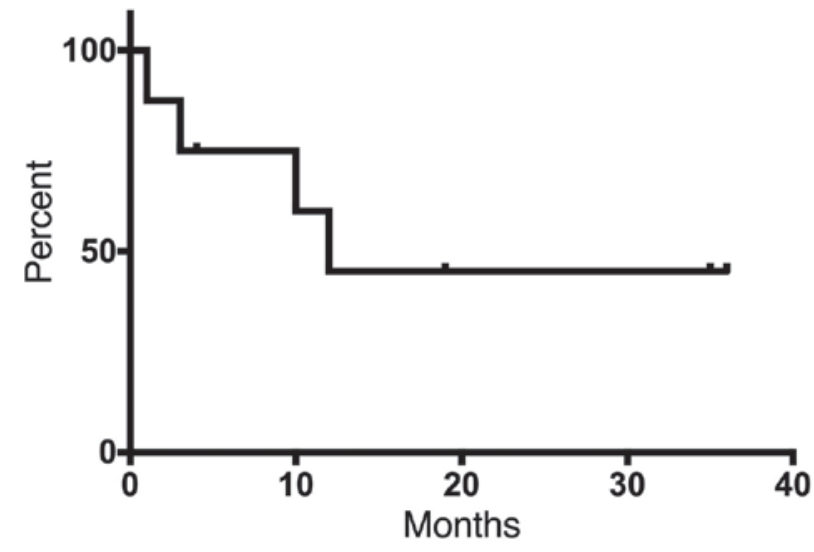

Figure 4. A total of 8 patients with a single viable disease were analyzed for disease-free survival (DFS). The median DFS was 12 months and the 12-month DFS rate was $45 \%$.

with previous reports, patients with a single viable tumor in our study exhibited significantly better survival following RT compared with those with multiple tumors.

Although experimental in vitro data have shown evidence of sarcoma radiosensitivity not different from that of epithelial neoplasms, sarcomas are considered as radioresistant tumors (16). RT is generally reserved for unresectable tumors and used primarily in the palliative setting. Kepka et al (9) reported that local control for unresectable sarcomas requires relatively high doses when delivered exclusively using external-beam RT. According to those findings, moderate doses of radiation in a conventional schedule were insufficient. Additional high-dose treatment approaches have been investigated, including intraoperative RT and brachytherapy $(7,8)$.

Linear energy transfer (LET), defined as the rate of energy loss from charged particles in tissue, is used to assess the biological effect of a particular radiation on the tissue. LET determines the biological impact of the energy deposited in the tissue. With an increase in LET, there is an initial increase in the relative biological effect. Higher LET radiation reduces the effects of tissue oxygenation and the sensitivity to variations in the cell cycle and DNA repair. For these reasons, using higher LET charged particles (e.g., carbon ion) for treatment improves local control $(17,18)$. In an experimental model of fibrosarcoma, Fukawa et al (19) reported that X-ray irradiation resulted in reduced reoxygenation and a heterogeneous oxygen partial pressure when compared with carbon-ion irradiation. As oxygen is an important factor in determining tumor response to RT, those data suggest that carbon-ion therapy may be superior to other forms of irradiation. Hypofractionated RT at higher doses per fraction may enhance radiation-induced tumor cell death by inducing endothelial apoptosis and subsequent microvascular damage $(20,21)$. Alternatively, modern RT modalities, including CyberKnife, IMRT and stereotactic RT (SRT), deliver photons and provide larger irradiated doses with OAR sparing when compared with conventional techniques. In the present study, we demonstrated that external-beam RT using photons improved local control rates with a high feasibility.

There were several limitations associated with this study. One limitation is the presentation of short-term outcomes in a limited number of patients. We confirmed the feasibility of 
RT for refractory sarcoma using modern X-ray-based RT techniques, including IMRT and SRT. Another limitation of our study design was that over half of our patients had received RT prior to this study. The time between pathological confirmation and RT varied in range among our study group. An extended follow-up may enable the analysis of slower-growing tumors, potentially strengthening the evidence for the efficacy of RT. The local control rate was excellent in this study, although the follow-up was not sufficiently long to evaluate local control or survival rate. Based on the present study, we consider that RT using modern techniques may significantly improve local control rates and, possibly, survival. As all the eligible patients had refractory sarcomas following initial treatment, local control was a primary outcome in our study. To investigate the efficacy and optimal protocol for RT delivered using photons, a prospective clinical trial with a long follow-up should be conducted.

Randomized, controlled clinical trials are well-established methods for providing the evidence necessary to design treatment plans. In previous controlled studies, aggressive treatment strategies for metastatic sarcoma were shown to improve survival $(5,6)$. Radical treatment techniques for local sites have significantly improved in recent decades. Improvements in radical treatment may enable effective management of unresectable refractory tumors. Unfortunately, a clinical study designed to directly compare the survival benefits between different modalities for treating refractory tumors would be difficult, due to variable patient history.

Targeted agents, including vascular endothelial growth factor inhibitors, were recently reported to show efficacy in sarcoma when combined with RT $(22,23)$. Doses $>10$ Gy likely produce secondary cell elimination due to enhanced vascular damage $(20,21)$. Compared with conventional schedules, we used relatively large doses per fraction, which potentially caused differences in biological reactions following radiation exposure. Further investigations are required to determine the optimal RT protocol and the efficacy of combined therapy using recently developed targeted agents and immunotherapy.

In conclusion, our study demonstrated that radical RT using modern techniques is highly feasible and may be an effective treatment option for refractory sarcoma.

\section{References}

1. National Comprehensive Cancer Network. NCCN Guidelines for Patients: Soft Tissue Sarcoma, version 1, 2014.

2. ESMO/European Sarcoma Network Working Group: Soft tissue and visceral sarcomas: ESMO Clinical Practice Guidelines for diagnosis, treatment and follow-up. Ann Oncol 25 (Suppl 3): iii102-iii112, 2014

3. Pisters PW, Leung DH, Woodruff J, Shi W and Brennan MF: Analysis of prognostic factors in 1,041 patients with localized soft tissue sarcomas of the extremities. J Clin Oncol 14: $1679-1689,1996$

4. Edmonson JH, Ryan LM, Blum RH, Brooks JS, Shiraki M, Frytak S and Parkinson DR: Randomized comparison of doxorubicin alone versus ifosfamide plus doxorubicin or mitomycin, doxorubicin, and cisplatin against advanced soft tissue sarcomas. J Clin Oncol 11: 1269-1275, 1993.

5. Billingsley KG, Burt ME, Jara E, Ginsberg RJ, Woodruff JM, Leung DH and Brennan MF: Pulmonary metastases from soft tissue sarcoma: Analysis of patterns of diseases and postmetastasis survival. Ann Surg 229: 602-610, discussion 610-612, 1999.
6. Falk AT, Moureau-Zabotto L, Ouali M, Penel N, Italiano A, Bay JO, Olivier T, Sunyach MP, Boudou-Roquette P, Salas S, et al; Groupe Sarcome Francais-Groupe D'etude Des Tumeurs Osseuses: Effect on survival of local ablative treatment of metastases from sarcomas: A study of the French Sarcoma Group. Clin Oncol (R Coll Radiol) 27: 48-55, 2015.

7. Koizumi M, Inoue T, Yamazaki H, Teshima T, Tanaka E, Yoshida K, Imai A, Shiomi H, Kagawa K, Araki N, et al: Perioperative fractionated high-dose rate brachytherapy for malignant bone and soft tissue tumors. Int J Radiat Oncol Biol Phys 43: 989-993, 1999.

8. Mahmoud $\mathrm{O}$ and Wolfson A: Perioperative irradiation in extremity soft tissue sarcoma. Expert Rev Anticancer Ther 11: 1233-1241, 2011.

9. Kepka L, DeLaney TF, Suit HD and Goldberg SI: Results of radiation therapy for unresected soft-tissue sarcomas. Int J Radiat Oncol Biol Phys 63: 852-859, 2005.

10. Ciernik IF, Niemierko A, Harmon DC, Kobayashi W, Chen YL, Yock TI, Ebb DH, Choy E, Raskin KA, Liebsch N, et al: Proton-based radiotherapy for unresectable or incompletely resected osteosarcoma. Cancer 117: 4522-4530, 2011.

11. Levine AM, Coleman C and Horasek S: Stereotactic radiosurgery for the treatment of primary sarcomas and sarcoma metastases of the spine. Neurosurgery 64 (Suppl 2): A54-A59, 2009.

12. Mehta N, Selch M, Wang PC, Federman N, Lee JM, Eilber FC, Chmielowski B, Agazaryan N, Steinberg M and Lee P: Safety and efficacy of stereotactic body radiation therapy in the treatment of pulmonary metastases from high grade sarcoma. Sarcoma 2013: 360214, 2013

13. Folkert MR, Bilsky MH, Tom AK, Oh JH, Alektiar KM, Laufer I, Tap WD and Yamada Y: Outcomes and toxicity for hypofractionated and single-fraction image-guided stereotactic radiosurgery for sarcomas metastasizing to the spine. Int J Radiat Oncol Biol Phys 88: 1085-1091, 2014.

14. Miura H, Inoue T, Shiomi H and Oh RJ: Differences in rates of radiation-induced true and false rib fractures after stereotactic body radiation therapy for stage I primary lung cancer. J Radiat Res 56: 332-337, 2015.

15. National Cancer Institute: Common Terminology Criteria for Adverse Events v3.0 (CTCAE). Available at:http://ctep.cancer. gov/protocolDevelopment/electronic_applications/docs/ctcaev3. pdf. Accessed September 19, 2015.

16. Gerweck LE, Zaidi ST and Zietman A: Multivariate determinants of radiocurability. I: Prediction of single fraction tumor control doses. Int J Radiat Oncol Biol Phys 29: 57-66, 1994.

17. Matsunobu A, Imai R, Kamada T, Imaizumi T, Tsuji H, Tsujii H, Shioyama Y, Honda H and Tatezaki S; Working Group for Bone and Soft Tissue Sarcomas: Impact of carbon ion radiotherapy for unresectable osteosarcoma of the trunk. Cancer 118: 4555-4563, 2012.

18. Jingu K, Tsujii H, Mizoe JE, et al; Organizing Committee for the Working Group for Head-and-Neck Cancer: Carbon ion radiation therapy improves the prognosis of unresectable adult bone and soft-tissue sarcoma of the head and neck. Int J Radiat Oncol Biol Phys 82: 2125-2131, 2012.

19. Fukawa T, Takematsu K, Oka K, Koike S, Ando K, Kobayashi H and Tanishita $\mathrm{K}$ : Differences in $\mathrm{pO}_{2}$ peaks of a murine fibrosarcoma between carbon-ion and X-ray irradiation. J Radiat Res 45: 303-308, 2004.

20. Park HJ, Griffin RJ, Hui S, Levitt SH and Song CW: Radiation-induced vascular damage in tumors: Implications of vascular damage in ablative hypofractionated radiotherapy (SBRT and SRS). Radiat Res 177: 311-327, 2012.

21. Brown JM, Carlson DJ and Brenner DJ: The tumor radiobiology of SRS and SBRT: Are more than the 5 Rs involved? Int J Radiat Oncol Biol Phys 88: 254-262, 2014.

22. Rao SS, Thompson C, Cheng J, Haimovitz-Friedman A, Powell SN, Fuks Z and Kolesnick RN: Axitinib sensitization of high single dose radiotherapy. Radiother Oncol 111: 88-93, 2014.

23. Lee HJ, Yoon C, Park DJ, Kim YJ, Schmidt B, Lee YJ, Tap WD, Eisinger-Mathason TS, Choy E, Kirsch DG, et al: Inhibition of vascular endothelial growth factor A and hypoxia-inducible factor $1 \alpha$ maximizes the effects of radiation in sarcoma mouse models through destruction of tumor vasculature. Int J Radiat Oncol Biol Phys 91: 621-630, 2015. 